\section{Frische Osteoporosefraktur: Vertebroplastie als wirksames Analgetikum}

\author{
Randomisierten kontrollierten Studien zufolge ist bei Fragilitätsfrakturen von \\ Wirbelkörpern, die bis zu zwölf Monate alt sind und immer noch Schmerzen \\ verursachen, von einer Vertebroplastie kein Nutzen zu erwarten. Anders sieht \\ es offenbar aus, wenn man das Verfahren bei frischen Frakturen anwendet.
}

Den primären Endpunkt, eine Schmerzreduktion auf NRS-Werte $<4$ am Tag 14 nach dem Eingriff, erreichten 24 Patienten $(44 \%)$ in der Verum- und $12(21 \%)$ in der Kontrollgruppe $(\mathrm{p}=0,011)$. Auch zu allen späteren Kontrollen bis zum Studienende nach sechs Monaten war die Rate von Vertebroplastie-Patienten mit NRS-Werten $<4$ durchweg größer als in der Vergleichsgruppe.

Ein Nutzen der PMMA-Injektion zeigte sich auch im Roland Morris Disability Questionnaire nach ein, drei und sechs Monaten sowie im Analgetikaverbrauch nach drei und sechs Monaten. Der Krankenhausaufenthalt war signifikant kürzer als nach dem Placeboeingriff (8,5 versus 14 Tage). Eine Subgruppenanalyse zum primären Endpunkt ergab, dass nur Patienten mit thorakolum- balen Frakturen von der Vertebroplastie profitierten (NRS $<4: 61 \%$ versus $13 \%$ ). Entscheidend für die Wirksamkeit der Vertebroplastie ist den Autoren zufolge, den Wirbelkörper mit ausreichend PMMA auszufüllen, um eine weiteren Einbruch zu verhindern. Das injizierte PMMA-Volumen sei in der Studie deutlich größer gewesen als in früheren randomisierten kontrollierten Studien.

Fazit: Patienten mit einer frischen (maximal sechs Wochen alten) Osteoporosebedingten thorakolumbalen Wirbelfraktur profitierten in der Studie von Clark et al. im Hinblick auf die Schmerzreduktion von einer Vertebroplastie. Der Vorteil bei diesem primären Endpunkt gegenüber einer Scheinbehandlung ist den Autoren zufolge zwar geringer ausgefallen als angestrebt. Mit einem absoluten Unterschied von $23 \%$ statt $30 \%$ war er aber immer statistisch signifikant.

Dr. Beate Schumacher

Clark W et al. Safety and efficacy of vertebroplasty for acute painful osteoporotic fractures (VAPOUR): a multicentre, randomised, doubleblind, placebo-controlled trial.

Lancet 2016; 388: 1408-16

\title{
Kniearthrose: Chondroitin und Glucosamin in Studie ohne gesicherten Nutzen
}

\section{Mangels krankheitsmodifizierender Alternativen und entgegen aller medizi- nischen Evidenz geben Patienten mit Gonarthrose in der Hoffnung auf Linderung ihres Leidens nach wie vor viel Geld für Chondroitin- und Glucosa- min-haltige Produkte aus. Eine placebokontrollierte Studie spanischer Ärzte konnte jedoch einmal mehr keine positiven Effekte nachweisen.}

V oraussetzung für die Teilnahme der Studie von Dr. Jorge Roman-Blas und Kollegen von der Fundación Jiménez Díaz in Madrid waren Kniegelenkschmerzen im Bereich von 40-80 Punkten auf einer 100-Punkte-Analogskala. Von 164 Arthrosepatienten bekam die Hälfte sechs Monate lang Placebo, die übrigen erhielten täglich $1.200 \mathrm{mg}$ hochreines Chondroitinsulfat aus Rinderknorpel zusammen mit $1.500 \mathrm{mg}$ kristallinem Glucosaminsulfat aus Krustentieren.

Im Lauf der Studie brachen $18 \%$ in der Placebo- und $31 \%$ in der Verumgruppe die Behandlung ab. Unter Chondroitin und Glucosamin wurden vermehrt Magen-Darm-Beschwerden beobachtet, etwa Durchfall, Verstopfung und Bauchschmerzen. Dies wirkte sich negativ auf die Ergebnisse aus. So war, bezogen auf sämtliche Teilnehmer, der Schmerz nach sechs Monaten in der Placebogruppe signifikant stärker zurückgegangen als unter Verum (minus 21 Punkte versus minus 12 Punkte). Wurden nur die Teilnehmer betrachtet, die bis zum Studienende durchhielten, gab es hingegen keine signifikanten Unterschiede, wenn- gleich die Schmerzlinderung unter Placebo auch hier tendenziell stärker war.

Ähnliches ließ sich mit einem anderen Tool beobachten, dem Western Ontario and McMaster Universities Osteoarthritis Index (WOMAC). Beim Gesamtscore und dem Schmerzscore schnitten die Patienten in der Intention-to-treat-Gruppe mit Placebo ebenfalls signifikant besser $a b$, wohingegen es bei der Per-ProtocolAnalyse keine statistisch belastbaren Unterschiede gab.

Fazit: In der placebokontrollierten Studie von Roman-Blas et al. konnte kein Nutzen von Chondroitin und Glucosamin in der Behandlung von Kniegelenkarthrosen nachgewiesen werden.

Thomas Müller

Roman-Blas JA et al. Chondroitin sulfate plus glucosamine sulfate shows no superiority over placebo in a randomized, double-blind, placebo-controlled clinical trial in patients with knee osteoarthritis. Arthritis \& Rheumatology; doi 10.1002/art.39819 